\title{
Primitive ontology and quantum field theory
}

\author{
Vincent Lam*
}

\begin{abstract}
Primitive ontology is a recently much discussed approach to the ontology of quantum theory according to which the theory is ultimately about entities in 3-dimensional space and their temporal evolution. This paper critically discusses the primitive ontologies that have been suggested within the Bohmian approach to quantum field theory in the light of the existence of unitarily inequivalent representations. These primitive ontologies rely either on a Fock space representation or a wave functional representation, which are strictly speaking unambiguously available only for free systems in flat spacetime. As a consequence, it is argued that they do not constitute fundamental ontologies for quantum field theory, in contrast to the case of the Bohmian approach to quantum mechanics.
\end{abstract}

\section{Introduction}

The aim of the primitive ontology (PO) approach to the interpretation of quantum mechanics (QM) is to provide a clear and realist ontology of the theory (i.e. a clear picture of what there fundamentally is in the world according to the theory) in terms of local material entities in 3-dimensional space (or 4-dimensional spacetime) and their temporal evolution. A PO can be specified in different ways, depending on what fundamental entities in 3-dimensional space are considered (particles, fields, strings, loops, ...). Despite all the controversies around the issue of the ontology of QM, it is a remarkable fact that the three standard realist interpretations of QM can be understood along the lines of the PO framework (which constitutes what Allori et al. 2008 identify as the "common structure" of these interpretations) - each actually specifying a different PO: point-like massive particles following continuous deterministic trajectories within the framework of Bohmian mechanics (BM), a continuous stochastic matter density field or stochastic point-like events ('flashes') within the theory of Ghirardi, Rimini and Weber (GRW), giving rise to the two versions GRWm and GRWf respectively. A primitive ontology in the Everett (or 'many-worlds') context can possibly be defined, for instance in terms of a deterministic matter density field (see Allori et al. 2008, §6.2), but its meaning is less transparent.

According to the PO proponents, such a 'common-sense' ontology for QM postulating from the start matter localized in 'usual' physical space or spacetime as the referent of the theory (i.e. as what QM is fundamentally about) is especially appealing for the powerful and simple explanatory scheme it allows. Indeed, within this framework, familiar macroscopic objects localized in 3-dimensional space and their (classical) behavior can be understood in terms of the behavior of (possibly fundamental) microscopic entities that are also localized in 3-dimensional space. In particular, there is an explicit connection between the behavior of these microscopic entities and what can be observed at the macroscopic level, for instance in terms of measurement outcomes. Obviously, the details of this account depend on the specific PO under consideration (e.g. particles, matter density field, flashes). The point is that such an account in terms of a PO does not have to bridge substantial explanatory gaps, for instance between macroscopic objects that are (or seem to be) localized in 3-dimensional space and fundamental microscopic entities that are not, as it seems to be the case within the framework of wave function realism for

* Department of Philosophy, University of Lausanne, CH-1015 Lausanne. E-mail: vincent.lam@unil.ch. 
instance, according to which QM is fundamentally about a wave function on a high-dimensional space.

The PO approach to QM finds part of its roots in Bell's notion of 'local beables', which was introduced in the context of his reflections on non-locality and the measurement problem (see the papers collected in Bell 1987). To some extent, a PO is made up of local beables - that is, of entities that "can be assigned to some bounded spacetime region" (Bell 1987, 53)—which can be directly related to the behavior of familiar material objects, such as a measurement apparatus for instance, so that the measurement problem simply does not arise within the framework of a PO for QM (hence the common structure shared by the standard realist interpretations of QM that provide a solution to the measurement problem).

In this context, it seems natural to try to extend the PO interpretive move to the (larger) domain of (relativistic) quantum field theory (QFT). Indeed, this latter theory is currently our best fundamental physical theory about matter and constitutes the theoretical background of the experimentally extremely successful Standard Model of high-energy physics. From a scientific realist perspective, QFT is therefore a natural candidate for serious ontological investigations about the fundamental nature of matter and the PO approach provides a natural context for such investigations.

The aim of this paper is to critically discuss this PO approach to the ontology of QFT in the light of the existence of unitarily inequivalent representations of the relevant algebraic structures encoding the canonical commutation relations. We briefly review the general Bohmian strategy for specifying a PO for QFT (section 2), before discussing concrete examples recently elaborated in the literature, where the PO is specified in terms particles (section 3) or, perhaps more surprisingly in the Bohmian context, in terms of fields (section 4). Then we introduce the relevant aspects of the foundational issue of unitarily inequivalent representations in QFT (section 5) and argue that they undermine the original interpretive project of the proposed POs for QFT (section 6). The conclusion of the paper highlights the fact that being serious about (physical) ontology requires being serious about physics too (section 7).

\section{Bohmian strategy for specifying the primitive ontology of QFT}

$\mathrm{BM}$ embodies the paradigmatic strategy for specifying a primitive ontology for QM; as such, the natural extension of this Bohmian strategy to QFT constitutes a very convenient study case for discussing the PO move in the QFT context. In this section we first expose the general features of this strategy, as well as the two main ways it can be implemented in QFT (as throughout the entire paper, we emphasize a qualitative discussion over the technical details).

Within the framework of BM, the primitive ontology is given by point-like material particles (Bohmian particles obviously are local beables since they always have a definite position in 3-dimensional physical space), which deterministically evolve along well-defined continuous trajectories according to the Bohmian law of motion or guiding equation

$$
\frac{\mathrm{d} Q(t)}{\mathrm{d} t}=\mathrm{v}_{t}{ }_{t}[Q(t)]
$$

where $Q(t)=\left(Q_{1}, \ldots, Q_{N}\right)$ is the configuration of the particle positions and the 'velocity' vector field $\mathrm{v}^{\psi_{t}}$ is a functional of the usual QM wave function $\psi_{t}$, which itself satisfies the standard Schrödinger equation (the detailed expression of $\mathrm{v}_{t}$ is not needed here, see Dürr and Teufel 2009 for the details on BM; let us highlight the fact that the dynamics of the Bohmian particles crucially relies on $\psi_{t}$, whose status has therefore to be clarified within the PO framework, see Belot 2012 for a recent overview of this issue). Although BM is a fully deterministic theory, its complete empirical equivalence with QM is guaranteed by the assumption - called quantum equilibrium hypothesis - that the Bohmian particles are distributed following Born's rule, i.e. the particle position probability density is equal to $\left|\psi_{t}\right|^{2}$, where the relevant effective wave function is considered (the quantum equilibrium hypothesis can be further analyzed in different 
ways, for instance in terms of a notion of 'typicality' for initial configurations). So, a welldefined quantum equilibrium distribution (which is preserved by the equivariance property of the dynamics) - indeed, a well-defined quantum equilibrium measure - together with well-defined guidance and Schrödinger equations constitute key ingredients of the Bohmian strategy for specifying a primitive ontology. But of course, the first and most important ingredient is the set of local beables one starts with.

Within the framework of QFT, there are two natural choices for local beables, corresponding to two distinct approaches to QFT, one in terms of a Fock space representation, the other in terms of a wave functional representation. The former naturally leads to an interpretation of the theory in terms of particles (or 'quanta'), i.e. to the choice of particles as local beables, whereas the latter suggests a PO of classical field configurations (the local beables being classical fields over bounded spacetime regions). These two distinct approaches to QFT lead to different POsone in terms of particles, the other in terms of fields - but both are being specified through the above described Bohmian strategy (applied to particles or fields). Indeed, the central point of this paper relies on the fact that there is a precise sense in which they are actually equivalent.

A note on the relativistic aspect of QFT: as is well-known, the just described Bohmian strategy is not Lorentz invariant and involves a preferred reference frame. However, quantum equilibrium ensures that the Bohmian models for QFT make the same predictions as 'relativistic' QFT (the quotation marks refer to the fact that predictions are made within what is called regularized QFT, which in general is not Poincaré invariant, see section 7).

Let us now consider the Bohmian particle and field ontologies in some more details.

\section{A primitive ontology of particles}

A Fock space representation for a free QFT system in Minkowski spacetime is generated by the quantization of the relevant free classical field in terms of what can be interpreted as particle annihilation and creation operators (resulting from an appropriate Fourier decomposition). According to the usual, textbook understanding of QFT, a crucial element of this particle interpretation is of course the existence of a total number operator and considerations about energy eigenvalues of the eigenstates of this operator provide further ground for this interpretation, so that these (specific) states can be naturally understood as corresponding to situations with $n$ (non-interacting) particles (with $n$ the eigenvalue of the number operator). However, there is no such obvious correspondence for most states in the relevant Hilbert space (called Fock space), where superpositions of $n$-particle states with different $n$ are ubiquitous. Similarly to the case of non-relativistic QM, making ontological sense of such quantum superpositions is precisely the aim of defining a PO and the Bohmian approach provides a clear and efficient way to achieve this. Indeed, the Bohmian approach to QFT in terms of particles takes particles ontologically seriously: at a given time, there is always a well-defined particle number, although it can vary with time through literal annihilation and creation of particles.

In order to get a rough idea of how it works, we now give a brief sketch of the model proposed by Dürr et al. (2004) for such a Bohmian QFT, which generalizes the original work of Bell. As within $\mathrm{BM}$, pointlike material particles in 3-dimensional space constitute the $\mathrm{PO}$ of the theory; their equation of motion is of the form $\frac{d Q(t)}{d t}=\mathrm{v}^{\Psi_{t}}[Q(t)]$, but unlike BM, it is interrupted at random times by stochastic jumps to a different sector of the total configuration space $\mathcal{Q}=$ $\bigcup_{n=0}^{\infty} \mathcal{Q}^{[n]}$, where $\mathcal{Q}^{[n]}$ is the $n$-particle configuration space (single particle species are considered for simplicity). The usual deterministic continuous trajectories of Bohmian particles can literally stop or begin at physical annihilation or creation events, so that the global dynamics is stochastic. This feature might be surprising at first in a Bohmian context, where determinism is often understood as a key aspect. However, it is less surprising if one remembers that the primary aim of the Bohmian approach is not to provide a deterministic picture of the world. Rather it aims to solve the measurement problem and to provide a clear and coherent - in particular, observer independent - ontology in the form of a PO for the theory under consideration. In the case of this Bohmian approach to QFT, we have a fundamental ontology of Bohmian particles - a definite 
number of them at any given time- - with a globally stochastic dynamics. This dynamics depends on the quantum state, for which the quantum equilibrium distribution and the Schrödinger equation can also be defined in this QFT context; as within BM, the ontological status of the quantum state needs to be clarified (see above section 2).

Before turning to the field ontology, let us note that Colin and Struyve (2007) propose an alternative deterministic model for Bohmian QFT in terms of a 'Dirac sea' particle ontology, where the actual positive energy particles move against the background of a sea of actual negative energy particles (the 'Dirac sea'). As a consequence, and avoiding all the technical subtleties involved in these models, it is interesting to note that Bohmian QFT seems to allow for both a stochastic and a deterministic PO of particles (as intuitively expected, the ontological status of anti-particles is also completely different in the two cases: substantial Bohmian particles in the former case, mere 'holes in the sea' in the latter case).

\section{A primitive ontology of fields}

The configuration space of QM, over which the wave function is defined, suggests what the local beables of BM are, namely pointlike particles with well-defined positions in 3-dimensional space. By analogy, since the wave functional is defined over classical field configurations within the framework of the wave functional representation of QFT, it is natural to take these classical field configurations as local beables. More precisely, in this field perspective, the local beables are (classical) fields with determinate field values at spacetime points or regions. In close analogy with BM, a Bohmian law of motion of the form $\frac{\partial \varphi(x, t)}{\partial t}=\mathrm{v}^{\Psi_{t}}[\varphi(x, t)]$ (in the case of a scalar field $\varphi$ ) defines their temporal development, with the wave functional (since it is defined over a space of fields) $\Psi_{t}[\varphi(x, t)]$ satisfying a functional Schrödinger equation (and such that the quantum equilibrium distribution is well-defined and preserved).

As a concrete example, let us briefly consider the case of the free quantized electromagnetic field, for which a Bohmian model was first proposed by Bohm himself (see Struyve 2010 for an excellent overview of the Bohmian approaches to QFT in terms of field beables). In this case, the PO is constituted by the transverse part of the vector potential $A^{T}$ (which is gauge independent), or, perhaps more intuitively, by the related magnetic or electric fields that can be defined from $A^{T}$. Guidance and Schrödinger equations together with an appropriate quantum equilibrium measure can be defined for these fields and the corresponding wave functional defined over them. So far, so good for the free electromagnetic field. But that alone does not seem to be enough to account for the whole material world that surrounds us and it seems necessary to introduce beables for the quantum fields corresponding to 'normal', fermionic matter. However, in the spirit of Bell's reflections on local beables with respect to spin, Struyve and Westman (2007) argue that the field beables for the electromagnetic field might actually be sufficient for such an account, in the sense that they "record and display outcomes of measurements and, more generally, contain an image of the everyday classical world" (3116), provided the relevant fermionic degrees of freedom correctly influence the dynamics though the wave functional.

Defining a field PO for fermionic quantum fields proves to be much harder. Several proposals for fermionic field beables have been suggested, for instance in terms of $S U(2)$ Euler angles or Grassmann fields, but it is hard to see how they relate to the fundamental intuition behind the whole PO move and its explanatory power, namely that of material entities localized in 3dimensional space (see section 1). In order to remedy this difficulty, Struyve and Westman (2007, $\S 5)$ suggest to add to the field beables of the electromagnetic field an energy or matter density

field defined in terms of the actual configuration of these beables and the wave functional (from an ontological point of view, such a matter density field is very much like the one of GRWm).

\section{Unitarily inequivalent representations}

The two sets of POs for QFT - one in terms of particle beables, the other in terms of field beables - discussed in the last two sections rely on two distinct representations of the relevant 
algebraic structures encoding the canonical commutation relations, the Fock space representation and the wave functional representation respectively. Even if they generate ontologically different POs, these two representations are actually physically equivalent in a precise sense discussed below (see section 6).

Within the framework of QFT, it happens that there actually are (infinitely) many unitarily inequivalent representations. This section describes the relevant aspects of the existence of such unitarily inequivalent representations in QFT - a crucial and distinctive feature of QFT and one of the most important issues in QFT. The next section addresses the consequences for the two sets of POs specified in the preceding sections. In keeping with the spirit of this paper, we privilege a qualitative discussion over the technical details (which have been extensively discussed in the philosophy of physics literature, see e.g. the recent monograph by Ruetsche 2011, which I roughly follow below and to which I refer for more).

A QFT can be understood as the result of the canonical quantization of a classical field theory, and this procedure involves defining a representation of the appropriate commutation relations, that is a Hilbert space $\mathcal{H}$ with bounded operators satisfying these relations. More technically, these latter can be understood in terms of an algebraic structure called a $C^{*}$ algebra, and a representation of a $C^{*}$-algebra $\mathcal{A}$ is then defined as a morphism $\pi: \mathcal{A} \rightarrow \mathcal{B}(\mathcal{H})$ from $\mathcal{A}$ into the algebra $\mathcal{B}(\mathcal{H})$ of bounded operator on a Hibert space $\mathcal{H}$. Two representations $\left(\pi_{1}, \mathcal{H}_{1}\right)$ and $\left(\pi_{2}, \mathcal{H}_{2}\right)$ of $\mathcal{A}$ are unitarily equivalent if and only if there is a one-to-one invertible norm-preserving linear map $U: \mathcal{H}_{1} \rightarrow \mathcal{H}_{2}$ such that $U \pi_{1}(A) U^{-1}=\pi_{2}(A), \forall A \in \mathcal{A}$. The important aspect here is that two unitarily equivalent representations of a $\mathrm{QFT}$ are commonly understood as being physically equivalent or more precisely as having the same empirical content as characterized by expectation values (however, they can be ontologically inequivalent, see below section 6). But even more importantly, unitarily inequivalent representations of QFT are in general understood as having different empirical content as characterized by expectation values and therefore as being physically inequivalent.

The crucial point is that the quantization of a classical field theory - that is, a theory with infinitely many degrees of freedom - in order to obtain a QFT leads to (infinitely) many unitarily inequivalent representations of the relevant algebra encoding the appropriate commutation relations (the Stone-von Neumann theorem that ensures unitary equivalence does not apply to systems with an infinite number of degrees of freedom). This means in principle that there are many physically inequivalent representations of a QFT in the sense of having different empirical contents (e.g. different assignments of expectation values). For instance, an inertial and an accelerating observer in Minkowski spacetime generate unitarily inequivalent Fock space representations, each defining different vacuum states and different total particle number operators, in particular such that the total particle number operator corresponding to one representation is not defined for the other. The two observers will in general not agree about the empirical (particle) content of a QFT defined in this context.

From a general point of view, there is no fundamental reason to privilege one representationmore precisely, one equivalence class of unitarily equivalent representations - over all the many others unitarily (and physically) inequivalent representations that are available. One may try to resist taking this plethora of inequivalent representations into account, by singling out a privileged equivalence class of representations on the basis of certain specific criteria such as (Minkowski) spacetime symmetries. However, such a move cannot constitute a solid ground for fundamental ontological investigations, since these latter would be reliable only to the extent that the invoked specific criteria remain valid (for instance, invoking the symmetries of Minkowski spacetime might be useful for practical purposes, but QFT aims at being studied in the more general context of curved spacetimes and ultimately in the case where spacetime itself is considered as a dynamical entity in its own right; in any case, it does not seem reasonable to restrict in principle fundamental ontological considerations to flat spacetime).

There actually is a strong positive argument in favor of taking seriously the existence of unitarily inequivalent representations for interpretive purposes. Indeed, they play a crucial role in understanding several fundamental physical features and mechanisms. The above discussed unitarily inequivalent representations corresponding to observers with different (inertial and 
accelerating) states of motion are central to the understating of - and the difficulties linked with - the Unruh effect (see e.g. Ruetsche 2011, chap. 9). Similarly, the existence of unitarily inequivalent representations is crucial to a satisfactory account of spontaneous symmetry breaking (unitarily inequivalent representations lead to multiple vacuum states breaking the relevant symmetry of the theory, see Earman 2004) and superselection sectors (unitarily inequivalent representations ground the partition of the representations into unitary equivalence classes at the basis of superselection sectors, see Earman 2008).

So, let us now consider what are the consequences of the existence of unitarily inequivalent representations for the proposed POs of QFT.

\section{Equivalent difficulties for particle and field primitive on- tologies}

The existence of unitarily inequivalent representations has been explicitly exploited in the philosophy of physics literature in order to argue against the notion of particle being fundamental in QFT, in particular in the cases involving an accelerating observer (Clifton and Halvorson 2001) or curved spacetime (Arageorgis et al. 2002). One should be careful when evaluating the consequences of these arguments for the particle PO elaborated in the context of the Bohmian approach to QFT. Indeed, in general, considerations relying on the standard formalism of quantum theory - e.g. considerations about operators - do not necessarily possess the same (ontological) meaning within the Bohmian framework (Daumer et al. 1997). By contrast, the very existence of unitarily inequivalent representations, including non-Fock space representations, clearly poses a challenge for Bohmian QFT in terms of particles since this latter is committed to a Fock space representation. This challenge can be nicely illustrated by considering interactions more carefully.

Indeed, Fraser (2008) convincingly argues that the Fock space representation is in general and strictly speaking only available for free, non-interacting QFT systems; central to her argument is the fact that free and interacting QFT systems involve unitarily inequivalent representations (this can also be seen as a consequence of Haag's theorem, see Earman and Fraser 2006). So, there is in general no Fock space representation for a QFT with interactions - that is, a realistic QFT. This fact poses a serious difficulty for the Bohmian particle ontology of QFT, since it crucially relies on the Fock space representation (see section 3). Indeed, this PO would hold only for free QFT, that is, would only specify what the free theory is about - what there is in the world according to the free theory. This is hardly satisfying for a fundamental ontology. In other terms, since the world clearly contain interacting fundamental physical systems that QFT aims to describe, it seems that the whole PO move in terms of particles as expressed within the Bohmian approach cannot even get off the ground. Even in the free context, distinct but related difficulties arise for the Bohmian particle ontology in the cases mentioned above involving unitarily inequivalent Fock space representations (accelerating observer, curved spacetime): in these cases, the challenge for the Bohmian approach is about privileging one specific representation.

In the face of the difficulties of the PO in terms of particles, one might expect that the POs in terms of fields would fare better. However, a few considerations quickly dampen this expectation. Indeed, it turns out that the wave functional representation, which underlines the field ontologies in the Bohmian approach (see section 4), is unitarily equivalent to a Fock space representation of free QFT, which underlines the particle ontology in the Bohmian approach, so that most of the difficulties faced by the latter infect the former (see Baker 2009). As a consequence of the above discussion about Fock space representation, there is in general no wave functional representation for a QFT with interactions. So the POs in terms of fields (within the Bohmian approach) cannot account for interacting QFT systems, but only for free, non-interacting ones; again, hardly satisfying as a fundamental ontology. And similarly to the particle case, even in the free context, these field POs have to face ambiguities about unitarily inequivalent wave functional representations when an accelerating observer or curved spacetime 
is involved.

\section{Conclusion}

As it stands, the Bohmian strategy for specifying the primitive ontology of QFT relying on the Fock space or the wave functional representations is unsatisfactory because it does not take into account the existence of unitarily inequivalent representations - one of the most important foundational issues in QFT. Relying on such a specific class of representations designed for free systems cannot ground an appropriate ontological picture of the real physical world (note that this difficulty concerns not only the Bohmian approach to QFT, but all the POs of QFT that rely on these representations; for instance in the GRW context, see Tumulka 2006).

But QFT makes predictions that are empirically extremely well-confirmed in real world experiments, obviously involving interactions. And it does so by (among other things) imposing various constraints so that the number of degrees of freedom becomes finite; indeed, an infinite number of degrees of freedom is at the root of the existence of inequivalent representations (see section 5) and the source of the divergence of various empirically relevant elements of the theory. These constraints typically involve various cutoffs at short ('ultra-violet') and long ('infra-red') length scales, together with sophisticated renormalization group methods (note that one important side effect is that the resulting regularized QFT is not Poincare invariant). Why couldn't a proponent of the Bohmian strategy for specifying a PO for QFT just rely on this framework - which is the standard framework for the vast majority of physicists working in the domain - thereby merely eschewing the issue of the existence of unitarily inequivalent representations? Indeed, that's exactly what they do (e.g. Dürr et al. 2004: "[...] we show how, with (more or less) any regularized quantum field theory (QFT), one can associate a particle theory - describing moving particles - that is empirically equivalent to that QFT.")

Investigating to what extent the Bohmian strategy for defining a quantum theory in a serious way ('without observer') can be extended to QFT is natural and highly interesting in its own right. And conducting such investigations within the framework of regularized QFT is fair enough. But one should be very careful when drawing ontological conclusions on this basis, in particular claims about the PO of the theory. The main reason is that regularized QFT is (at) best considered as an effective theory, so that ontological considerations on this basis can only be effective in the same way. To be clear: the point here is not to argue along the lines of some pessimistic meta-induction argument, this would be an entirely different debate. Without discussing the status of regularized QFT and related cutoff and renormalization procedures (this would be another debate too), the point is rather that these latter completely leave out of the picture one of the most fundamental features of QFT, namely the existence of unitarily inequivalent representations. This is arguably a legitimate move for practical purposes (e.g. calculating cross-sections). But disregarding such a fundamental feature of QFT cannot provide the best ontology for this theory (or at least, it should be explicitly argued why a primitive ontologist for QFT is entitled to disregard the issue of inequivalent representations, in particular in the face of their alleged physical significance). The early Bohmians and later the proponents of the PO approach to QM urged that we should be serious about the theory and its ontology; coherence would require the same attitude for QFT.

Acknowledgements I am grateful to the Swiss National Science Foundation (Ambizione grant PZ00P1_142536/1) for financial support. 


\section{References}

Allori, Valia, Sheldon Goldstein, Roderich Tumulka, and Nino Zanghì. 2008. "On the Common Structure of Bohmian Mechanics and the Ghirardi-Rimini-Weber Theory." British Journal for the Philosophy of Science 59: 353-389.

Arageorgis, Aristidis, John Earman, and Laura Ruetsche. 2002. "Weyling the time away: the non-unitary implementability of quantum field dynamics on curved spacetime." Studies in History and Philosophy of Modern Physics 33: 151-184.

Baker, David John. 2009. "Against Field Interpretations of Quantum Field Theory." British Journal for the Philosophy of Science 60: 585-609.

Bell, John. S. 1987. Speakable and Unspeakable in Quantum Mechanics. Cambridge: Cambridge University Press.

Belot, Gordon. 2012. "Quantum states for primitive ontologists." European Journal for Philosophy of Science 2: 67-83.

Clifton, Rob, and Hans Halvorson. 2001. "Are Rindler Quanta Real? Inequivalent Particle Concepts in Quantum Field Theory." British Journal for the Philosophy of Science 52: 417470.

Colin, Samuel, and Ward Stuyve. 2007. "A Dirac sea pilot-wave model for quantum field theory." Journal of Physics A 40: 7309-7342.

Daumer, Martin, Detlef Dürr, Sheldon Goldstein, and Nino Zanghì. 1997. "Naive realism about operators." Erkenntnis 45: 379-397.

Dürr, Detlef, and Stefan Teufel. 2009. Bohmian Mechanics. Dordrecht: Springer.

Dürr, Detlef, Sheldon Goldstein, Roderich Tumulka, and Nino Zanghì. 2004. "Bohmian Mechanics and Quantum Field Theory." Physical Review Letters 93: 090402.

Earman, John. 2004. "Curie's Principle and spontaneous symmetry breaking." International Studies in the Philosophy of Science 18: 173-198.

Earman, John. 2008. "Superselection Rules for Philosophers." Erkenntnis 69: 377-414.

Earman, John, and Doreen Fraser. 2006. "Haag's theorem and its implications for the foundations of quantum field theory." Erkenntnis 64: 305-344.

Fraser, Doreen. 2008. "The fate of 'particle' in quantum field theories with interactions." Studies in History and Philosophy of Modern Physics 39: 841-859.

Ruestche, Laura. 2011. Interpreting Quantum Theories. Oxford: Oxford University Press.

Struyve, Ward. 2010. "Pilot-wave theory and quantum fields." Reports on Progress in Physics 73: 106001.

Struyve, Ward, and Hans Westman. 2007. "A minimalist pilot-wave model for quantum electrodynamics." Proceedings of the Royal Society A 463: 3115-3129.

Tumulka, Roderich. 2006. "On spontaneous wave function collapse and quantum field theory." Proceedings of the Royal Society A 462: 1897-1908. 